\title{
UNA REVISIÓN DE ESCALAS DE EVALUACIÓN PARA MEDIR EL CAMBIO DE CONDUCTA DEBIDO A LA LESIÓN CEREBRAL Y EL TRATAMIENTO DE ESTOS CAMBIOS \\ A REVIEW OF RATING SCALES FOR MEASURING BEHAVIOR CHANGE DUE TO BRAIN INJURY AND TREATMENT FOR THESE CHANGES
}

\author{
Natalia Martín de la Huerga, Vega Muriel, Celeste Aparicio-López, Rocío \\ SÁnCHEZ-CARrión y TERESA RoIg Rovira
}

Cómo referenciar este artículo/How to reference this article:

Martín de la Huerga, N., Muriel, V., Aparicio-López, C., Sánchez-Carrión, R. y Roig Rovira, T. (2014). Una revisión de escalas de evaluación para medir el cambio de conducta debido a la lesión cerebral y el tratamiento de estos cambios [A Review of Rating Scales for Measuring Behavior Change Due To Brain Injury and Treatment for These Changes]. Acción Psicológica, 11(1), 79-94. http://dx.doi. org/10.5944/ap.1.1.13878

\section{Resumen}

Introducción: Las personas que han sufrido una lesión cerebral suelen experimentar un amplio conjunto de alteraciones cognitivas, emocionales y conductuales. La rehabilitación neuropsicológica acostumbra a centrarse en los aspectos cognitivos, dando escasa o nula atención al resto de cambios. En este contexto los cambios conductuales son un gran hándicap para la rehabilitación y la adaptación a la sociedad. Objetivo: Revisar los hallazgos obtenidos en estudios sobre escalas que evalúan los cambios de conducta en lesión cerebral y apliquen tratamientos específicos. Método: La revisión incluyó artículos originales que utilizaban algún cuestionario y tratamiento para valorar y mejorar los cambios conductuales en adultos que habían sufrido una lesión cerebral. Se consultaron las bases de datos CINAHL y MEDLINE entre los años 1990 a 2014. Se seleccionaron instrumentos utilizados en población adulta con lesión cerebral. Se excluyeron escalas psiquiátricas o aplicadas a población sin daño cerebral. Resultados: La búsqueda produjo un gran número de referencias y se identificaron varios instrumentos. La mayoría cuantificaban los cambios conductuales, pero además en muchos de ellos se abordan aspectos emocionales, psiquiátricos o de calidad de vida. La mayoría de las escalas se auto-administraban tanto al paciente como al familiar o cuidador. Conclusiones: A pesar de la poca variedad de escalas dedicadas a la evaluación de la conducta, se encontraron instrumentos idóneos en la práctica clínica para el diagnóstico de cambios

Correspondencia: Institut Guttmann, Institut Universitario de Neurorrehabilitación, afiliado a la Universidad Autónoma de Barcelona. 
conductuales. Respecto al tratamiento, la literatura describe estudios de caso único y grupal; sin embargo, no se considera que exista un tratamiento único para los pacientes con lesión cerebral.

Palabras clave: tratamiento; escala; lesión cerebral; conducta; neuropsicología.

\section{Abstract}

Introduction: People who have suffered a brain injury often experience a wide range of cognitive, emotional and behavioural disorders. Neuropsychological rehabilitation used to focus on the cognitive aspects, giving little or no attention to the other changes. In this context the behavioural changes are a big handicap for their rehabilitation and adaptation to society. Aim: To review the findings obtained in studies on scales assessing behavioural changes in acquired brain injury (ABI) and to apply specific treatments. Method: The review included original articles and treatments, which used questionnaires to assess and improve the behavioural changes in adults who had suffered an ABI. CINAHL and MEDLINE databases between the years 1990-2014 were consulted. Instruments used in adults with DCA were selected. Psychiatric scales applied to people without brain damage were excluded. Results: The search yielded a large number of references and several instruments were identified. Most quantified behavioural changes, but also many emotional, psychiatric or quality of life issues are addressed. Most self-administered scales assess both the patient and the family or caregiver. Conclusions: Despite the limited range of scales involved in the assessment of behaviour, suitable tools were found in clinical practice for the diagnosis of behavioral changes. Regarding treatment, the literature describes studies of single and group cases, however, no single treatment for patients with DCA is identified.

Keywords: treatment; scale; acquired brain injury; behavior; neuropsychology.

\section{Introducción}

En los últimos años ha tenido lugar un progresivo incremento de personas afectadas por lesión cerebral, en concreto de Accidente Cerebrovascular o ictus, al contrario que los Traumatismos Craneoencefalicos (TCE) que han disminuido. Los ictus afectan entre 120000 y 130000 de personas al año en España y son la segunda causa de muerte en varones y la primera en mujeres. Estos trastornos comportan cambios físicos, cognitivos, emocionales y conductuales en la persona afectada que, a su vez, repercuten en la familia y el entorno -ver Figura 1- (Brooks y McKinlay, 1983; Marsh y Kersel, 2006; Perlesz, Kinsella, y Crowe, 1999).

La persona afectada con una lesión cerebral ve un cambio drástico de su vida, ya que sufre alteraciones físicas (motoras y sensitivas) y neuropsicológicas (cognitivas, conductuales $\mathrm{y}$ emocionales). Las alteraciones cognitivas más comunes son los déficits de atención, memoria o disfunción ejecutiva; siendo la depresión y la ansiedad, las manifestaciones más típicas emocionalmente. En cuanto a la conducta, puede observarse un cambio en la personalidad de estos pacientes aumentando las manifestaciones de agitación, irritabilidad, agresividad, desinhibición, apatía, egocentrismo, depresión y ansiedad. 


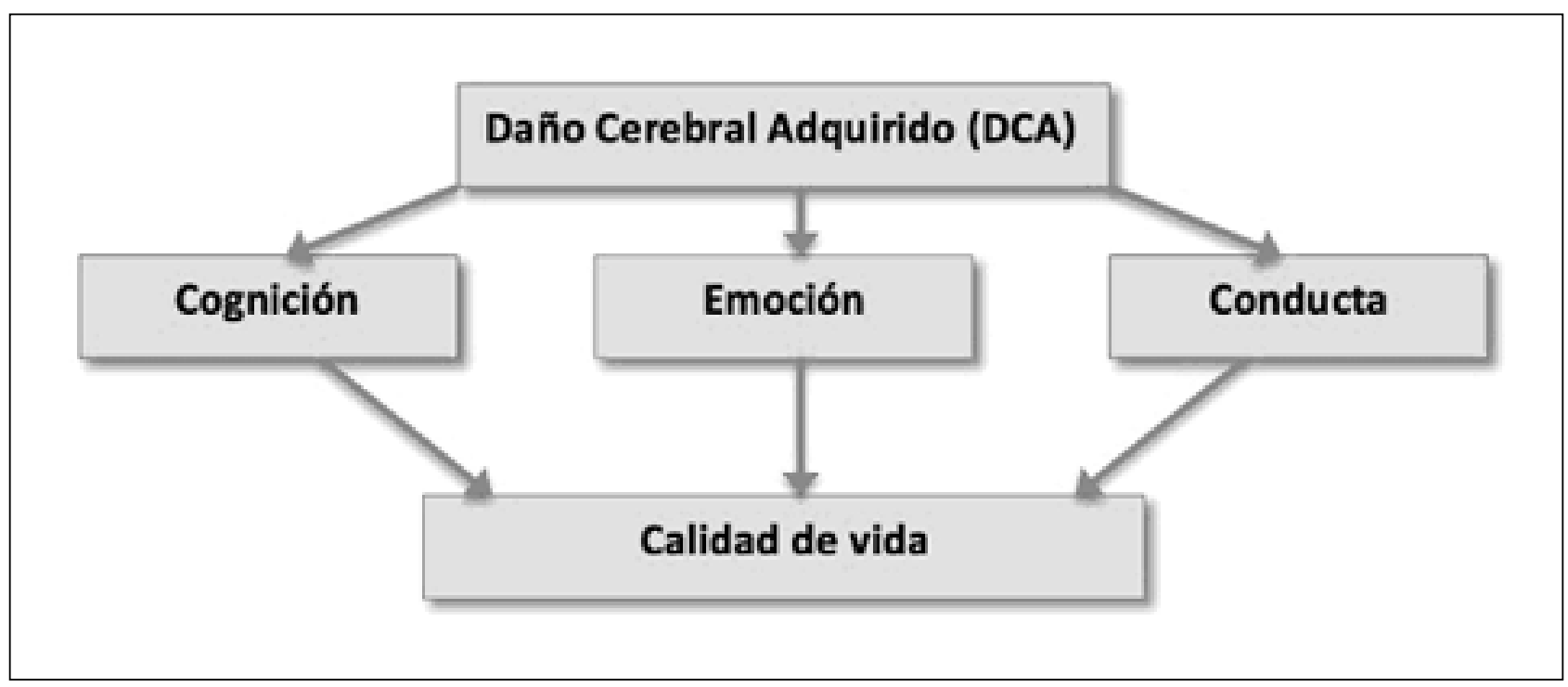

Figura 1. Variables afectadas tras la lesión cerebral

Los cambios conductuales se producen entre el 40 y $80 \%$ de los pacientes con lesión cerebral y son problemas que, sin un tratamiento específico y adecuado persisten a lo largo del tiempo e incluso tienden a empeorar si no se realiza una intervención específica (McLean, Temkin, y Dikmen, 1984).

A pesar de la gran incidencia de estos cambios e impacto que producen, no hay un gran abanico de posibles escalas para su cuantificación en comparación con la amplia oferta de test y escalas existentes para otros déficits, como son los cognitivos o emocionales. Esto supone un problema ya que no hay una adecuada evaluación, por su escasez de pruebas e importantes dificultades metodológicas; como la variabilidad de la sintomatología a lo largo del tiempo, las limitaciones de conocer la personalidad premórbida, la escasa conciencia de los déficits, o la falta de adecuación de los instrumentos entre otros. Asimismo esto se une al hecho de que no existe una fundamentación teórica sólida lo que dificulta la existencia de protocolos de evaluación (Sánchez-Cubillo, Lertxundi, Quemada, Ruiz-Ruiz, 2007).

Además del impacto en el entorno familiar, los déficits conductuales implican un hándicap en el proceso rehabilitador, éste se ve interferido en gran medida por la presencia de este tipo de comportamientos. Por ello es de gran utilidad enmarcar la situación actual del paciente tras sufrir la lesión cerebral, evaluar qué cambios se han producido y cómo repercute a su vida diaria para poder realizar un tratamiento adecuado y adaptado al paciente y sus necesidades.

El objetivo de este artículo ha sido revisar los estudios que se han realizado en la neuropsicología, buscando escalas de evaluación de conducta y respectivos tratamientos.

\section{Método}

\section{Estrategia de búsqueda}

La estrategia de búsqueda fue diseñada para recuperar el mayor número de referencias relacionadas con los cambios y tratamientos conductuales tras lesión cerebral mediante escalas y terapias. Para ello han utilizado las siguientes palabras clave (brain injury, adquired Brain injury, traumatic brain injury, ictus, behavior, behavioral changes, treatment, scales, evaluation).

Se consultó en CINAHL y MEDLINE por ser dos bases de datos que indexan un gran porcentaje de producción mundial de artículos en las ciencias de la salud. El periodo de búsqueda se planteó entre los años 1980 y 2014, lo suficientemente amplio para confiar en que la información recuperada sea relevante. 


\section{Procedimiento de selección}

Se incluyeron artículos originales que utilizaban cuestionarios o terapias para valorar y tratar los cambios conductuales en lesión cerebral.

Los artículos elegidos cumplían los siguientes criterios de inclusión:

- Estudiaban cuestionarios centrados en la conducta tras una lesión cerebral.

- Planteaban tratamientos de los déficits conductuales en pacientes que han sufrido una lesión cerebral.

- Y eran artículos con una de la edad muestral igual o superior a 18 años.

Como criterios de exclusión se decidió que los artículos tenían que estar centrados en la conducta, y no en variables psiquiátricas.

\section{Aspectos conceptuales}

Para comprender cómo se interpretan las alteraciones conductuales en lesión cerebral es importante considerar el origen y la evolución del concepto. Tras la descripción clásica del paciente Phineas Cage por John M. Harlow a mediados del siglo XIX (Harlow, 1848, 1868), comenzaron a aparecer trabajos en los que se explicaban cambios de personalidad y de la conducta de los pacientes como consecuencia de lesiones cerebrales. En especial del lóbulo frontal y temporal, ambos muy sensibles a la lesión cerebral. La afectación de estas áreas puede producir la ruptura del sistema límbico estrechamente vinculado al procesamiento emocional, motivacional y conductual (Warriner y Velikonja, 2006).

Por lo tanto, una clasificación clásica de los trastornos de conducta según la afectación del sistema dañado es la siguiente (ver Figura 2):

- Sistema frontal: desinhibición, euforia, descenso de tacto social, comportamiento pueril.

- Sistema subcortical: apatía, descenso de vitalidad, impulsividad y falta de espontaneidad

- Sistema temporal: irritabilidad y agresividad.

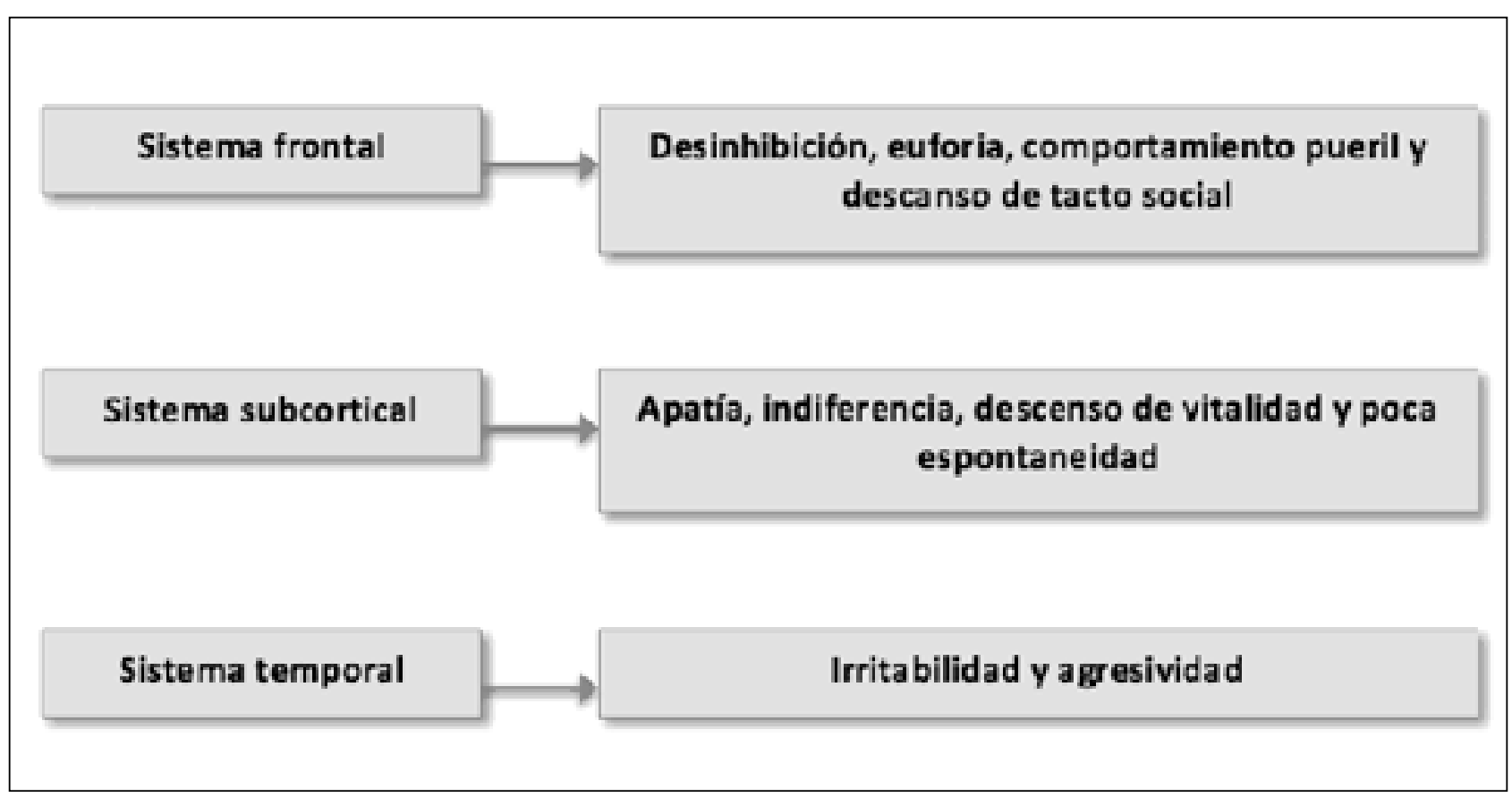

Figura 2. Clasificación de afectación de sistemas y sintomatología 
Autores como Wood, Alderman, Warriner y Cummings entre otros han realizado trabajos interesantes sobre la anatomía, casos de lesión cerebral y alteración de la conducta y descripción de manifestaciones clínicas como agresividad, comportamiento pueril, irritabilidad, depresión, ansiedad o falta de habilidades sociales, sin embargo, la visión actual se dirige a una visión más global del cerebro y no al localizacionismo obsoleto (Duffau, 2012).

\section{Resultados}

\section{Escalas y cuestionarios}

De la revisión de escalas las más nombradas en la literatura son las siguientes (ver Tabla 1):

Patient Competency Rating Scale (PCRS) desarrollada por George Prigatano y colaboradores en 1986. El objetivo es evaluar la capacidad de las propias fortalezas y debilidades tras la lesión cerebral, a partir de 30 ítems con una escala de tipo Likert de 1 a 5, donde se preguntan diferentes actividades de la vida diaria. Evalúa diferentes ámbitos: calidad de vida, conducta, emoción, cognición y capacidad física. Existen tres versiones: paciente, cuidador y clínico.

Se han realizado tres estudios transculturales (Prigatano y Leathem, 1993; Prigatano, 1997, 1998). Además de tres versiones: inglesa, japonesa y española; y dos estudios normativos Prigatano (1988) y Leathem (1998). Existe una versión reducida Patient Competency Rating Scale-Neurorehabilitation (PCRS-NR) realizada por Borgado y Prigatano en 2003, para pacientes en rehabilitación hospitalaria.

La fiabilidad de la escala es de 0.97 para los pacientes y 0.92 para los familiares (Prigatano, Altman y O’Brien, 1990), la consistencia interna también es alta en pacientes y familiares, con un alfa de Cronbach de 0.91 y de 0.93 respectivamente.

Neurobehavioral Functioning Inventory (NFI) desarrollada por Kreutzer, Seel, y Marwitz en 1997. Su objetivo es evaluar los síntomas conductuales y emocionales tras el lesión cerebral. Utiliza una escala de tipo Likert de 1 a 5. Analiza temas frecuentes de la vida diaria, problemas emocionales y cambios conductuales. Existen dos versiones: paciente y familiar, ambas con contenido idéntico.

La NFI se añadió al protocolo de seguimiento de pacientes con lesión cerebral en National Institute on Disability and Rehabiltation Research (NIDRR). Los propios autores de la escala remarcaron que la concordancia entre la respuesta del paciente y el familiar debe verse respecto a otras variables como la edad, gravedad de la lesión o la relación entre el familiar y paciente. También se realizó un estudio con pacientes con TCE donde se observó que la NFI diferenciaba entre pacientes depresivos y no depresivos (Kennedy et al., 2005).

Los análisis estadísticos confirman una alta fiabilidad interna entre las versiones de las dos escalas, con un alfa de Cronbach de 0.86 y 0.95 respectivamente.

Overt Behaviour Scale (OBS) fue desarrollada por Gleen Kelly en 2006. El objetivo fue evaluar el comportamiento problemático tras la lesión cerebral, a partir de una entrevista semiestructurada de 34 ítems con nueve categorías. Se registran tres aspectos: los cambios producidos tras la lesión cerebral y su gravedad (agresividad, comportamiento antisocial, desinhibición, etc.), la frecuencia en que se producen estas manifestaciones; y el impacto de estos cambios producidos en el familiar, es decir, estrés emocional o carga familiar. Se creó una versión que se realiza al familiar o cuidador del paciente.

La fiabilidad, validez y capacidad de respuesta de la OBS se obtuvo a partir de una muestra de pacientes con lesión cerebral $(\mathrm{n}=$ 58) obteniendo una alta fiabilidad y estabilidad, de 0.97 y 0.77 respectivamente.

Self Awareness Of Déficits Interview (SADI) fue desarrollada por Fleming, Strong y Ashton en 1996. El objetivo fue obtener datos cuantitativos y cualitativos del estado de conciencia de los propios déficits y la habilidad para planificar objetivos realistas, mediante una entrevista semiestructurada. Esta escala presenta dos versiones: una para el paciente y otra para 
el familiar o cuidador. Está compuesta por tres ámbitos: déficit, funcionalidad y habilidad para crear objetivos realistas. En la primera parte de cada ámbito se realizan varias preguntas y a continuación se puntúa en una escala de tipo Likert de 0 a 3 la situación global del paciente en ese ámbito.

En el análisis estadístico se observó una alta fiabilidad se demostró con una consistencia test retest demostrada tanto para el resultado total de la escala ( $\mathrm{ICC}=94)$ como para las puntuaciones de las diferentes subescalas ( ICC $=0.85$, 0.86 , y 0.86). Además estos resultados combinados con investigaciones previas indican una alta fiabilidad interevaluador del SADI.

Self Regulation Skills Interview Ownsworth (SRSI) fue desarrollada por Tamara en 2000. El objetivo es conocer las habilidades metacognitivas del paciente, habilidades de autorregulación, la motivación, y el uso de estrategias de compensación. Está compuesta por una entrevista semiestructurada formada por seis ítems que evalúan la metacognición y la autorregulación. Se puntúa mediante una escala de tipo Likert de 0 a 10.

Estadísticamente los autores demostraron que la escala tenía una buena fiabilidad interevaluador (0.81-0.92) y test retest (0.69-0.91).

Wise (2005) utilizó esta prueba para realizar un estudio con pacientes que habían sufrido TCE y analizar la reinserción en el ámbito laboral.

Mayo Portland Adaptability Inventory (MPAI 4) fue desarrollada por Malec en 2005. El objetivo fue evaluar las alteraciones conductuales y sociales a largo plazo tras la lesión cerebral a partir de 219 ítems con una escala de tipo Likert de 0 a 4 , donde se preguntan diferentes cuestiones como el grado de ansiedad, las dificultades de memoria o concentración, fatiga o autocuidado; las cuales se engloban en tres índices: capacidad, ajuste y participación.

Hay varias versiones: ingles, francés, danés, español, alemán, italiano, portugués, sueco y holandés.
Estadísticamente la consistencia es excelente con una puntuación 0.78 a 0.99 , y una validez aceptable de 0.49 a 0.65 .

Neuropsychology Behavior And Affect Profile (NBAP) desarrollada por Nelson, Satz y D’Elia en 1994. El objetivo fue analizar los cambios de personalidad antes y después de la lesión cerebral. Primeramente se desarrolló para pacientes con demencia, pero se amplió a pacientes neurológicos, incluyendo ictus y TCE (Nelson, Drebing, Satz y Uchiyama, 1998). Consta de 106 ítems, donde se responde sí o no según las características del paciente (por ejemplo, «mi familiar es excesivamente hablador»). Está compuesta por cinco escalas que miden un constructo diferente: indiferencia, comportamiento inapropiado, depresión, manía y pragnosia (déficit de la comunicación pragmática). Sólo existe una versión, la del familiar o cuidador.

Estadísticamente presenta una buena consistencia interna de manía, depresión indiferencia con una alfa Cronbach superior a 0.70, de 0.79 en conducta y 0.49 para pragnosia. No obstante, encontraron una buena consistencia interna de todas las escalas de 0.76 .

Neurobehavioral Rating Scale Revised (NRS-R) desarrollada por Levin en 1987. Se desarrolló a partir de la Brief Psyquyatric Rating Scale (BPRS) que estudiaba las manifestaciones conductuales tras el periodo de Amnesia Postraumática (APT) tras el daño cerebral. Aunque esta escala evalúa aspectos conductuales también se centraba en los psiquiátricos, por lo que los autores realizaron una adaptación, la NRS- R. El objetivo es evaluar los cambios conductuales tras la lesión cerebral, a partir de 27 ítems, que integran diferentes tipos de cambio, como la desinhibición, apatía, etc. Está compuesta por una entrevista semiestructurada con una escala tipo Likert de 0 a 7 . Hay una versión para el familiar o cuidador.

Katz Adjustment Scale Form Revised (KAS R) desarrollada por Goran y Fabiano en 1993, es una adaptación de la Katz Adjustment Scale (KAS; Katz y Liverly, 1963). El objetivo fue evaluar los síntomas conductuales y emocionales, a partir de 79 ítems que se puntúan en una escala de tipo Likert de 1 a 4, los cuales incluyen aspectos como agresividad, negativismo, im- 
pulsividad, hiperactividad, entre otros. Existen dos versiones: paciente y familiar o cuidador.

Estadísticamente los autores demostraron una alta consistencia interna (alfa de Cronbach de 0.78 a 0.94).

The Frontal System Behavior Scale (FrSBe) desarrollada por Grace en 2001. El objetivo fue diagnosticar cambios de personalidad y conductuales tras una lesión en el lóbulo frontal, a partir de 46 ítems. Realiza una clasificación dependiendo de las manifestaciones en: apatía, desinhibición o disfunción ejecutiva. Existen dos versiones: paciente y familiar o cuidador.

Se han realizado diferentes estudios con población adicta a tóxicos (Miller et al., 2013), o pacientes con TCE e ictus (Caracuel et al., 2012).

Estadísticamente posee una alta consistencia interna demostrada por los propios autores, con un coeficiente de 0.92 y 0.88 para la versión paciente y familiar respectivamente.

Iowa rating scale of personality change (IRSPC) desarrollada por Barrash en 1993. Estos autores crearon esta escala tras la investigación donde se demostró la poca fiabilidad y validez de las pruebas específicas de personalidad en pacientes con daño cerebral. El objetivo fue evaluar los cambios afectivos, conductuales y sociales tras la lesión cerebral, a partir de 27 ítems. Se identifican, en primer lugar, las manifestaciones clínicas y en segundo lugar, el nivel de cambio. Existen dos versiones: paciente $\mathrm{y}$ familiar o cuidador.
Estadísticamente tiene una fiabilidad interevaluador alta de 0.80 y 0.96 tanto para la manifestación como para la puntuación del cambio.

Head Injury Behaviour Rating Scale (HIBS) desarrollada por Godfrey en 2003. Estos autores crearon esta escala autoadministrable para identificar los cambios emocionales y conductuales tras el TCE, creando así dos factores: Regulación Emocional y Regulación Conductual, en 20 ítems, con una escala tipo Likert de 1 a 4 . Existen dos versiones: paciente y cuidador o familiar.

Estadísticamente los resultados revelan una alta fiabilidad y validez, con un alfa de Cronbach de 0.91 y 0.87 para la regulación emocional y regulación conductual, respectivamente. La correlación entre estas dos subescalas es 0.65 .

Estudios interesantes sobre las alteraciones conductuales en pacientes que sufrieron el daño cerebral hace un año, como el de Marsh y Kersel (2006) demuestran la gran aportación de datos que se obtienen a partir de la HIBS.

En 2013, se realizó una validación de esta escala al castellano (López de Arroyabe, Calvete, Las Hayas y Zubizarreta, 2013), obteniendo una alta consistencia con un coeficiente de alfa de 0.94, 0.77 y 0.81, para la Regulación Emocional, Regulación Conductual y Problemas Psicológicos, respectivamente. El alfa total fue de 0.83 . 
Tabla 1.

Escalas de evaluación para medir el cambio de conducta debido a la lesión cerebral (elaboración propia).

\begin{tabular}{|c|c|c|c|c|c|c|}
\hline Escala & Autor & Año & $\begin{array}{l}\text { Ámbitos de } \\
\text { evaluación }\end{array}$ & $\begin{array}{l}\text { Tipo } \\
\text { de escala }\end{array}$ & Versiones & $\begin{array}{l}\text { Datos } \\
\text { normativos } \\
\text { en español }\end{array}$ \\
\hline $\begin{array}{l}\text { Patient Competency Rating } \\
\text { Scale (PCRS) }\end{array}$ & Prigatano & 1986 & $\begin{array}{l}\text { Emoción } \\
\text { Conducta } \\
\text { Cognición } \\
\text { Calidad de vida } \\
\text { Aspectos físico }\end{array}$ & Cuestionario & $\begin{array}{l}\text { Paciente } \\
\text { familiar o } \\
\text { cuidador } \\
\text { clínico }\end{array}$ & Sí \\
\hline $\begin{array}{l}\text { Neurobehavioral Rating Scale } \\
\text { Revised (NRS-R) }\end{array}$ & Levin & 1987 & $\begin{array}{l}\text { Emoción } \\
\text { Conducta }\end{array}$ & $\begin{array}{l}\text { Entrevista } \\
\text { semiestructu- } \\
\text { rada }\end{array}$ & $\begin{array}{l}\text { Familiar o } \\
\text { cuidador }\end{array}$ & Sí \\
\hline $\begin{array}{l}\text { lowa rating scale of personality } \\
\text { change (IRSPC) }\end{array}$ & Barrash & 1993 & $\begin{array}{l}\text { Conducta } \\
\text { Cognición }\end{array}$ & Cuestionario & $\begin{array}{l}\text { Paciente } \\
\text { familiar o } \\
\text { cuidador }\end{array}$ & No \\
\hline $\begin{array}{l}\text { Katz Adjustment Scale Form } \\
\text { Revised (KAS R) }\end{array}$ & $\begin{array}{l}\text { Goran y } \\
\text { Fabiano }\end{array}$ & 1993 & $\begin{array}{l}\text { Emoción } \\
\text { Conducta }\end{array}$ & Cuestionario & $\begin{array}{l}\text { Paciente } \\
\text { familiar o } \\
\text { cuidador }\end{array}$ & Sí \\
\hline $\begin{array}{l}\text { Neuropsychology Behavior And } \\
\text { Affect Profile (NBAP) }\end{array}$ & $\begin{array}{l}\text { Nelson, Satz y } \\
\text { D’Elia }\end{array}$ & 1994 & $\begin{array}{l}\text { Emoción } \\
\text { Conducta }\end{array}$ & Cuestionario & $\begin{array}{l}\text { Paciente } \\
\text { familiar o } \\
\text { cuidador }\end{array}$ & No \\
\hline $\begin{array}{l}\text { Self Awareness Of Déficits } \\
\text { Interview (SADI) }\end{array}$ & $\begin{array}{l}\text { Fleming, Strong } \\
\text { y Ashton }\end{array}$ & 1996 & $\begin{array}{l}\text { Emoción } \\
\text { Conducta }\end{array}$ & $\begin{array}{l}\text { Entrevista } \\
\text { semiestructu- } \\
\text { rada }\end{array}$ & $\begin{array}{l}\text { Paciente } \\
\text { Familiar o } \\
\text { cuidador }\end{array}$ & No \\
\hline $\begin{array}{l}\text { Neurobehavioral Functioning } \\
\text { Inventory (NFI) }\end{array}$ & $\begin{array}{l}\text { Kreutzer, Seel, } \\
\text { y Marwitz }\end{array}$ & 1997 & $\begin{array}{l}\text { Emoción } \\
\text { Conducta }\end{array}$ & Cuestionario & $\begin{array}{l}\text { Paciente } \\
\text { familiar o } \\
\text { cuidador }\end{array}$ & Sí \\
\hline $\begin{array}{l}\text { Self Regulation Skills Interview } \\
\text { Ownsworth (SRSI) }\end{array}$ & Ownswoth & 2000 & $\begin{array}{l}\text { Emoción } \\
\text { Conducta }\end{array}$ & $\begin{array}{l}\text { Entrevista } \\
\text { semiestructu- } \\
\text { rada } \\
\text { y cuestionario }\end{array}$ & Paciente & No \\
\hline $\begin{array}{l}\text { The Frontal System Behavior } \\
\text { Scale (FrSBe) }\end{array}$ & Grace & 2001 & $\begin{array}{l}\text { Conducta } \\
\text { Cognición }\end{array}$ & Cuestionario & $\begin{array}{l}\text { Paciente } \\
\text { familiar o } \\
\text { cuidador }\end{array}$ & Sí \\
\hline $\begin{array}{l}\text { Head Injury Behaviour Rating } \\
\text { Scale (HIBS) }\end{array}$ & Godfrey & 2003 & $\begin{array}{l}\text { Emoción } \\
\text { Conducta }\end{array}$ & Cuestionario & $\begin{array}{l}\text { Paciente } \\
\text { familiar o } \\
\text { cuidador }\end{array}$ & Sí \\
\hline $\begin{array}{l}\text { Mayo Portland Adaptability } \\
\text { Inventory (MPAI 4) }\end{array}$ & Malec & 2005 & $\begin{array}{l}\text { Emoción } \\
\text { Conducta } \\
\text { Cognición }\end{array}$ & Cuestionario & Paciente & Sí \\
\hline Overt Bahaviour Scale (OBS) & Kelly & 2006 & $\begin{array}{l}\text { Emoción } \\
\text { Conducta }\end{array}$ & $\begin{array}{l}\text { Entrevista } \\
\text { semiestructu- } \\
\text { rada }\end{array}$ & $\begin{array}{l}\text { Familiar o } \\
\text { cuidador }\end{array}$ & No \\
\hline
\end{tabular}




\section{Tipos de tratamiento}

En la literatura no existe un modelo consensuado para el tratamiento de los cambios conductuales en personas que hayan sufrido una lesión cerebral. Existen algunos autores que se centran en el tratamiento grupal, y otros en el individual.

El objetivo final como se ha comentado con anterioridad, sea tratamiento individual o grupal, es trabajar desde un modelo de rehabilitación holístico. Diversos estudios indican, que la inclusión de una actividad de psicoterapia (grupal o individual) dentro de un programa de rehabilitación es determinante para una mayor eficacia en el tratamiento, provocando a su vez una mayor independencia funcional y una reducción en el desajuste psicosocial y la incidencia de conductas desadaptativas (BenYshay y Diller, 1993; Cicerone, 2000, 2005).

Es muy importante saber los objetivos que se esperan alcanzar con el tratamiento y cuáles son los pasos que se deben dar. Para que el paciente tenga en cuenta la situación que está viviendo y la afronte de la manera más apropiada a partir de los objetivos marcados (Prigatano, 1999). Es necesario tener en cuenta que los pacientes con lesión cerebral atraviesan una serie de fases desde el momento del diagnóstico hasta la aceptación de la realidad: negación, ira, negociación, depresión y aceptación y que esto puede influir en el desarrollo del tratamiento.

En 1984 Prigatano creó uno de los primeros tratamientos específicos para cambios conductuales en pacientes con lesión cerebral desarrollando un programa de rehabilitación que establecía dos grupos: el tratamiento (experimental) y el grupo sin tratamiento (control). Los resultados indicaron que el grupo experimental mostró una mejoría significativa en dichos cambios, en la exploración neuropsicológica y una reducción del estrés emocional en comparación con el grupo control.

El momento más adecuado para comenzar con el tratamiento es en la fase ambulatoria, ya que el paciente comienza a tener un mayor grado de conciencia de sí mismo y de sus dificultades.

A pesar de las ventajas del tratamiento, hay que tener en cuenta que no todos los pacientes se podrán beneficiar en el mismo grado, debido a las alteraciones cognitivas y emocionales, que también repercuten en la evolución. Algunos autores reflejan la dificultad de la psicoterapia en daño cerebral por diferentes limitaciones, como los déficits de memoria (Judd y Wilson, 2005).

Las corrientes psicoterapéuticas más adecuadas para la intervención con personas con daño cerebral, pueden englobarse dentro de las Terapias Cognitivo Conductual (TCC) o humanistas, ya que se centran en la persona y en su desarrollo. A diferencia de terapias de tipo dinámico, que son muy prolongadas en el tiempo, y requieren capacidades que este tipo de pacientes puede tener dificultad para realizar (introspección o abstracción).

Dentro de las técnicas de las TCC se incluye el condicionamiento clásico, el condicionamiento operante y el aprendizaje vicario. Todos ellos analizan y manipulan la relación entre estímulos y respuestas de los sujetos, por medio de estímulos reforzantes o aversivos, basándose en la teoría de aprendizaje. Este tipo de técnicas es lo que se conoce como modificación de conducta y se utiliza en el ámbito de la neuropsicología como intervención de las alteraciones conductuales. La teoría de aprendizaje se basa en la comprensión de la relación entre el comportamiento desafiante y las contingencias ambientales, adaptándose a los déficits cognitivos. Ha sido utilizada tanto de forma individual como grupal, con el objetivo de manejar el comportamiento inapropiado después de la lesión cerebral (Giles y Manchester, 2006; Wood y Alderman, 2011). Existen otros dos tipos de tratamientos: Operant Neurobehavioral Approach (ONA) y el Relational Neurobehavioral Approach (RNA), el primero caracterizado por la utilización de refuerzos y el segundo por la influencia de la psicología social, el asesoramiento y la motivación (Gordon, Cantor, Ashman y Brown, 2006). La efectividad de este tipo de tratamiento se ha demostrado en recientes revisiones donde se 
aplican métodos operantes para el manejo de las alteraciones conductuales (Yvisaker et al., 2007; Wood y Alderman, 2011)

Algunos autores como Ownswoth, Alderman, Klimczak o Williams han aplicado diferentes tipos de tratamientos TCC individual describiendo casos únicos. No existen estudios que comparen la efectividad de los tipos de intervención psicoterapéutica, pero sí que se ha observado que ésta mejora la aceptación de la discapacidad, la capacidad de conciencia, una mejor adaptación y desarrollo de la persona en su nueva vida (Cicerone, 1989; Koetzer 2007; Prigatano, 1997; Wilson 2002). La falta de evidencia de la eficacia de la psicoterapia no se debe a su ineficacia sino a su difícil metodología.

Existen otros dos tipos de enfoque: el neuroconductual y la remediación cognitiva. El enfoque neuroconductual (Malhotra, Rajender, Sharma y Singh, 2009) se centra en el diagnóstico de alteraciones neurológicas y neuropsicológicas que interfieren en el aprendizaje y conducta del sujeto para crear un tratamiento basado en aspectos como en métodos operantes y orientación conductual. Mientras la remediación cognitiva (RC) creada por el King College de Londres para reforzar la capacidad neuropsicológica. Se ha empleado para mejorar desde aspectos cognitivos, conductuales como emocionales.

La psicoterapia grupal ofrece trabajar los aspectos conductuales y emocionales de forma idónea, ya que permite el apoyo entre los pacientes, el aprendizaje a través de las experiencias del resto de compañeros, el contraste de percepciones propias con el resto, favoreciendo así la evolución de la psicoterapia. StalderLüthy (2013) ha descrito un estudio reciente e interesante para trabajar la depresión en pacientes con daño cerebral. Recientemente autores como Azulay, Johansson o Bédard crearon programas de reducción del estrés basados en mindfulness en pacientes con TCE, teniendo como objetivo adaptarse a la nueva vida. Otros estudios realizados con TCC en lesión cerebral demuestran la efectividad de mejorar el estado de ánimo, la adaptación y la integración a la comunidad (Arundine et al., 2012).

\section{Discusión}

El estudio de la neuropsicología engloba la cognición, emoción y conducta. Por ello, deben tenerse en cuenta las tres en el momento de la rehabilitación e intervención tras una lesión cerebral. El objetivo de este artículo ha sido describir las escalas de evaluación de la conducta y describir de manera concisa los principios básicos del tratamiento en pacientes con déficits conductuales cuya presencia en los programas de rehabilitación neuropsicológica es escasa.

Autores como Prigatano, Goldstein o Wilson han remarcado que el objetivo de la rehabilitación en daño cerebral es ayudar al paciente a aceptar sus limitaciones y ayudarles a mejorar los cambios que han sufrido en su personalidad. La mezcla de psicoterapia, rehabilitación cognitiva y modificación de conducta son necesarias para la intervención de los problemas conductuales (Ylvisaker, 2002). No se puede separar de la rehabilitación neuropsicológica cognitiva, la conductual y la emocional ya que estas tres son un conjunto que va unido y que se interrelaciona. Este tipo de rehabilitación holística, centrada en el individuo, promueve una mejor rehabilitación y calidad de vida en pacientes con lesión cerebral.

El objetivo de la rehabilitación neuropsicológica no es solo mover la recuperación cognitiva de las funciones alteradas, sino tratar de modificar las conductas problemáticas. Para ello es muy importante trabajar con todo el equipo rehabilitador y sobretodo con la familia, ya que ésta podrá trabajar como coterapeuta. Es muy importante facilitar a los familiares herramientas para trabajar estos aspectos.

Dos aspectos que dificultan la evaluación de la conducta tras la lesión cerebral son la poca cantidad de este tipo de instrumentos y su objetivo de evaluación, ya que se suelen centrar más en aspectos emocionales.

De las escalas anteriormente señaladas, se concluyó como mejor herramienta la escala HIBS por su sencillez y validez, centrándose en aspectos emocionales y conductuales, y pu- 
diéndose comparar los dos tipos de versiones (paciente y familiar o cuidador).

Por otra parte, a pesar de la incidencia de estos problemas conductuales y el gran impacto que provocan en el entorno del paciente, no es un tema muy estudiado. Como se ha podido observar a lo largo de la revisión, la intervención en la lesión cerebral se ha centrado más en los déficits cognitivos pasando a un segundo plano los problemas conductuales que aparecen frecuentemente. Es por ello que debemos abrir más el foco de investigación hacia estos problemas que causan un gran impacto en la vida del propio individuo y en el entorno social para su posterior reinserción en la sociedad y entorno familiar.

\section{Referencias bibliográficas}

Alderman, N. (2003). Contemporary approaches to the managementof irritability and aggression following traumatic brain injury. Neuropsychological Rehabilitation, 13, 211-240.

Arundine, A., Bradbury, C. L., Dupuis, K., Dawson, D. R., Ruttan, L. A. y Green, R. E. (2012). Cognitive behavior therapy after acquired brain injury: maintenance of therapeutic benefits at 6 months posttreatment. Journal Head Trauma Rehabilitation, 27(2), 104-112.

Azulay, J., Smart, C. M., Mott, T. y Cicerone, K. D. (2013). A pilot study examining the effect of mindfulness-based stress reduction on symptoms of chronic mild traumatic brain injury/ postconcussive syndrome. The Journal of Head Trauma Rehabilitation, 28(4), 323-231.

Barrash, J., Tranel, D. y Anderson, S. W. (2000). Acquired personality disturbances associated with bilateral damage to the ventromedial prefrontal region. Developmental Neuropsychology, 18, 355-381.

Bédard, M., Felteau, M., Marshall, S., Cullen, N., Gibbons, C., Dubois, S.,... Moustgaard, A. (en prensa). Mindfulness-Based Cognitive Therapy Reduces Symptoms of Depression in People With a Traumatic Brain Injury: Results From a Randomized Controlled Trial. The Journal of Head Trauma Rehabilitation.
Bédard, M., Felteau, M., Marshall, S., Dubois, S., Gibbons, C., Klein, R. y Weaver, B. (2012). Mindfulness-based cognitive therapy: benefits in reducing depression following a traumatic brain injury. Advances in Mind Body Medecine, 26(1), 14-20.

Ben-Yshay, Diller L. (1993). Cognitive remediation in traumatic brain injury: update and issues. Archives of Physical Medicine and Rehabilitation, 74, 204-213.

Bigelow, H. J. (1850). Dr. Harlow's case of recovery from the passage of an iron bar through the head. The American Journal of the Medical Science, 20, 13-22.

Block, C. K. y West, S. E. (2013). Psychotherapeutic treatment of survivors of traumatic brain injury: review of the literature and special considerations. Brain Injury, 27(7-8), 775-88.

Borgaro, S. R. y Prigatano, G. P. (2003). Modification of the Patient Competency Rating Scale for use on an acute neurorehabilitation unit: the PCRS-NR. Brain Injury, 17 (10), 847-53.

Bowie, C. R., Gupta. M. y Holshausen, K. (2013). Cognitive remediation therapy for mood disorders: rationale, early evidence, and future directions. Canadian Journal of Psychiatry, 58(6), 319-325.

Bradbury, C. L., Christensen, B. K., Lau, M. A., Ruttan, L. A., Arundine, A. L. y Green, R. E. (2008). The efficacy of cognitive behavior therapy in the treatment of emotional distress after acquired brain injury. Archives of Physical Medicine and Rehabilitation, 89(12), 61-68.

Brooks, N., Campsie, L., Symington, C., Beattie, A. y McKinlay, W. (1986). The five year outcome of severe blunt head injury: a relative's view. Journal of Neurology, Nuerosurgery and Psychiatry, 49(7), 764-770.

Butler, R. W. y Satz, P. (1988). Individual psychotherapy with head injured adults: clinical notes for the practitioner. Proffesional Psychology: Research and Practice, 19, 536-541.

Caracuel, A., Verdejo, A., Fernández, M. J., Moreno, L., Santago, S., Salinas-Sánchez, I. y Pérez, M. (2012). Preliminary validation of the Spanish version of the Frontal Systems Behavior Scale (FrSBe) using Rasch analysis. Brain Injury, 26(6), 844-852. 
Cicerone, K. D. (1989). Psychotherapeutic interventions with traumatically brain injured patients. Rehabilitation Psychology, 34, 105-114.

Devany, C. W., Kreutzer, J., Halberstadt, L. y West, D. D. (1991). Referrals for supported employment after brain injury: Neuropsychological, behavioral, and emotional characteristics. Journal of Head Trauma Rehabilitation, 6(3), 59-70.

Duffau, H. (2012). The "frontal syndrome» revisited: Lessons from electrostimulation mapping studies. Cortex, 48(1),120-131.

Dvorkin, A., Pacini, S., Hsu, N. y Larson, E. (2013). Treatment challenges with profound behaviour disturbance after traumatic brain injury: A case report. Brain Injury, 27(7-8), 957-961.

Fabiano, R. J. y Goran, D. A. (1992). A principle component analysis of the Katz Adjustment Scale in a traumatic brain injury rehabilitation sample. Rehabilitation Psychology, 37, 75-85.

Fitzgerald, P. B., Hoy, K. E., Maller, J. J., Herring, S., Segrave, R., McQueen, S.,... Daskalakis, Z. J. (2011). Transcranial magnetic stimulation for depression after a traumatic brain injury: a case study. The Journal of ECT, 27(1), 38-40.

Fleming, J. M., Strong, J. y Ashton, R. (1996). Selfawareness of deficits in adults with traumatic brain injury: How best to measure? Brain Injury, 10, 1-15.

Fleming, J. M., Strong, J. y Ashton, R. (1998). Cluster analysis of self-awareness levels in adults with traumatic brain injury and relationship to outcome. Journal of Head Trauma Rehabilitation, 13, 39-51.

Giles, G. M. y Manchester, D. (2006). Two approaches to behavior disorder after traumatic brain injury. Journal of Head Trauma Rehabilitation, 21(2), 168-178.

Godfrey, H. P. D., Partridge, F. M., Knight, R. G. y Bishara, S. (1993). Course of insight disorder and emotional dysfunction following closed head injury: A controlled cross-sectional followup study. Journal of Clinical and Experimental Neuropsychology, 15, 503-515.

Goran, D. A. y Fabiano, R. J. (1993). The scaling of the Katz Adjustment Scale in a traumatic brain injury rehabilitation sample. Brain Injury, 7, 219-229.
Gordon, W. A., Zafonte, R., Cicerone, K., Cantor, J., Brown, M., Lombard L., Chandna T. (2006). Traumatic brain injury rehabilitation: state of the science. American Journal of Physical Medicine \& Rehabilitation, 85(4), 343-382.

Gordon, W. A., Cantor, J., Ashman T. y Brown, M. (2006). Treatment of post-TBI executive dysfunction: application of theory to clinical practice. The Journal of Head Trauma Rehabilitation, 21(2), 156-167.

Harlow, J. M. (1848). Passage of an iron rod through the head. Boston Medical and Surgical Journal, 39, 389 - 393.

Harlow, J. M. (1868). Recovery from the passage of an iron bar through the head. Publications of the Massachussets Medical Society, 2, 327-247.

Heilbronner, R. L., Millsaps, C., Azrin, R. y Mittenberg, W. (1993). Psychometric properties of the Patient Competency Rating Scale (PCRS). Journal of Clinical and Experimental Neuropsychology, 15, 67-68.

Hsieh, M. Y., Ponsford, J., Wong, D., Schönberger, M., McKay, A. y Haines, K. (2012). Development of a motivational interviewing programme as a prelude to CBT for anxiety following traumatic brain injury. Neuropsychological Rehabilitation, 22(4), 563-584.

Hufford, B. J., Williams, M. K., Malec, J. F. y Cravotta, D. (2012). Use of behavioural contracting to increase adherence with rehabilitation treatments on an inpatient brain injury unit: A case report. Brain Injury, 26 13), 1743-1749.

Johansson, B., Bjuhr, H. y Rönnbäck, L. (2012). Mindfulness-based stress reduction (MBSR) improves long-term mental fatigue after stroke or traumatic brain injury. Brain Injury, 26(13-14), 1621-1268.

Judd, D. y Wilson, S. L. (2005). Psychotherapy with brain injury survivors: an investigation of the challenges encountered by clinicians and their modifications to therapeutic practice. Brain Injury, 19, 437 449 .

Katz, M. M. y Lyerly, S. B. (1963). Methods for measuring adjustment and social behavior in the community: Rationale, description, discriminative validity, and scale development. Psychological Reports, 13, 503-535. 
Kean, J., Malec, J. F., Cooper, D. B. y Bowles, A. O. (2005). The Mayo Portland Adaptability Inventory. The Center for Outcome Measurement in Brain Injury. Archives of Physical Medicine and Rehabilotation, 94(12), 2417-2424

Kelly, G., Brown, S., Todd, J. y Kremer, P. (2008). Challenging behaviour profiles of people with acquired brain injury living in community settings. Brain Inury, 22(6), 457-470.

Kelly, G., Todd, J., Simpson, G., Kremer, P. y Martin, C. (2006). The overt behaviour scale (OBS): A tool for measuring challenging behaviours following ABI in community settings. Brain Injury, 20(3), 307-319.

Kennedy, R. E., Livingston, L., Riddick, A., Marwitz, J. H., Kreutzer, J. S. y Zasler, N. D. (2005). Evaluation of the Neurobehavioral Functioning Inventory as a depression screening tool after traumatic brain injury. Journal of Head Trauma Rehabilitation, 20(6), 512-526.

Kertesz, A., Davidson, W. y Fox, H. (1997). Frontal behavioral inventory: diagnostic criteria for frontal lobe dementia. Canadian Jorunal of Neurological Sciences. 24(1), 29-36.

Klimczak, N. J., Donovick, P. J. y Burright, R. G. (1997). Psychotherapy with the brain injured adult. Psychotherapy in Private Practice, 16, 33-44.

Koetzer, R. (2007). Psychotherapy Following Traumatic Brain Injury: Integrating Theory and Practice. Journal of Head Trauma Rehabilitation, 22(1), 39-47.

Krause, M., Mahant, N., Kotschet, K., Fung, V. S., Vagg, D., Wong, C. H. y Morris, J. G. (2012). Dysexecutive behaviour following deep brain lesions a different type of disconnection syndrome? Cortex, 48(1), 97-119.

Kreutzer, J. S., Marwitz, J. H., Seel, R. y Serio, C. D. (1996). Validation of a neurobehavioral functioning inventory for adults with traumatic brain injury. Archives of Physical Medicine and Rehabilotation, 77(2), 116-124.

Kreutzer, J., Devany, C., Myers, S. y Marwitz, J. (1991). Neurobehavioral outcome following traumatic brain injury: Implications for cognitive rehabilitation. Baltimore: Paul Brookes.

Leathem, J. M., Murphy, L. J. y Flett, R. A. (1998). Self and informant ratings on the Patient Competency Rating Scale in patients with traumatic brain injury. Journal of Clinical and Experimental Neuropsychology, 20, 694-705.

Levin, H. S., High, W. M., Goethe, K. E., Sisson, R. A., Overall, J. E. y Rhodes, H. M. (1987). The neurobehavioral rating scale: assessment of the behavioural sequelae of head injury by the clinician. Journal of Neurology, Neurosurgery and Psychiatry, 50, 183-193

Malhotra, S., Rajender, G., Sharma, V. Singh, T. B. (2009). Neurobehavioral management of behavioral anomalies in frontal lobe syndrome. Indian Journal of Psychological Medecine, 31(2), 98-100.

Malloy, P; Grace, J. (2005). A Review of Rating Scales for Measuring Behavior Change Due to Frontal Systems Damage. Cognitive and Behavioral Neurology, 18(1), 18-27.

Marsh, N. V. y Kersel, D. A. (2006). Frequency of behavioural problems at one year following traumatic brain injury: correspondence between patient and caregiver reports. Neuropsychological Rehabilitation, 16(6), 684-694.

McLean, A. J., Temkin, N. R., y Dikmen, S. (1983). The behavioral sequelae of head injury. Journal of Clinical and Experimental Neuropsychology 5, 361-376.

Miller, S. C., Baktash, S. H., Webb, T. S., Whitehead, C. R., Maynard, C., Wells, T. S.,... Gore, R. K. (2013). Risk for addiction-related disorders following mild traumatic brain injury in a large cohort of active-duty U. S. airmen. The American Journal of Psychiatry, 170(4), 383-390.

Nadell, J. (1991). Towards an existential psychotherapy with the traumatically brain injured adult. Cognitive Rehabilitation, 9, 8-13.

Nelson, L. D., Drebing, C., Satz, P. y Uchiyama, C. (1998). Personality change in head trauma: a validity study of the Neuropsychology Behavior and Affect Profile. Archives of Clinical Neuropsychology, 13(6), 549-560.

Ownsworth, T. L. (2005). The impact of defensive denial upon adjustment following traumatic brain injury. Neuropsychoanalysis, 7, 83-94.

Ownsworth, T. L., McFarland, K. M. y Young, R. M. (2000). Development and standardization of the Self-regulation Skills Interview (SRSI): a new clinical assessment tool for acquired brain injury. Clinical Neuropsychology. 14(1), 76-92. 
Ownsworth, T. L., McFarland, K. y Ross, Mc. D. Y. (2000). Development and Standardization of the Self-Regulation Skills Interview (SRSI), A New Clinical Assessment Tool for Acquired Brain Injury. The Clinical Neuropsychologist, 14,(1), 76-92.

Perlesz, A., Kinsella, G., y Crowe, S. (1999). Impact of traumatic Brain injury on the family: a critical review. Rehabilitation Psychology, 44(1), 6-35.

Prigatano, G. P. (1986). Neuropsychological rehabilitation after brain injury. Baltimore: Johns Hopkins University Press.

Prigatano, G. P. (1996). Behavioral limitations TBI patients tend to underestimate: A replication and extension to patients with lateralized cerebral dysfunction. Clinical Neuropsychologist, 10, 191-201.

Prigatano, G. P. (1997). Learning from our successes and failures: reflections and comments on "Cognitive rehabilitation: how it is and how it might be». Journal of the International Neuropsychological Society, 3(5), 497-499.

Prigatano, G. P. (1999). Principles of neuropsychological rehabilitation. New York: Oxford University Press

Prigatano, G. P. y Altman, I. M. (1990). Impaired awareness of behavioral limitations after traumatic brain injury. Archives of Physical Medicine and Rehabilitation, 71, 1058-1064.

Prigatano, G. P. y Leathem, J. M. (1993). Awareness of behavioral limitations after traumatic brain injury: A crossultural study of New Zealand Maoris and non-Maoris. Clinical Neuropsychologist, 7, 123-135.

Prigatano, G. P. y Leathem, J. M. (1993). Awareness of behavioral limitations after traumatic brain injury: A cross-cultural study of New Zealand Maoris and non-Maoris. Clinical Neuropsychologist, 7, 123-135.

Prigatano, G. P., Altman, I. M. y O'Brien, K. P. (1990). Behavioral limitations that brain injured patients tend to underestimate. Clinical Neuropsychologist, 4, 163-176.

Prigatano, G. P., Bruna, O., Mataro, M., Muñoz, J. M., Fernández, S. y Junque, C. (1998). Initial disturbances of consciousness and resultant impaired awareness in Spanish patients with brain injury. Journal of Head Trauma Rehabilitation, 13, 29-38.
Prigatano, G. P., Fordyce, D. J., Zeiner, H. K., Rouche, J. R., y Pepping, M. (1984). Neuropsychological rehabilitation after closed head injury in young adults. Journal of Neurology, Neurosurgy and Psychiatry, 47, 505-513.

Prigatano, G. P., Klonoff P., O’Bryen, K. P., Altman, I. M. y Chiapello, D. (1994). Productivity after neuropsychologically oriented milieu rehabilitation. Journal of Head Trauma Rehabilitation, 9, 91-102.

Prigatano, G. P., Ogano, M. y Amakusa, B. (1997). A cross-cultural study on impaired self-awareness in Japanese patients with brain dysfunction. Neuropsychiatry, Neuropsychology, and Behavioral Neurology, 10, 135-143.

Ranseen, J. D., Bohaska, L. A. y Schmitt, F. A. (1990). An investigation of anosognosia following traumatic brain injury. International Journal of Clinical Neuropsychology, 7, 29-36

Ruff, R. (2013). Selecting the appropriate psychotherapies for individuals with traumatic brain injury: what works and what does not? NeuroRehabilitation, 32(4), 771-779.

Sánchez-Cubillo, I., Lertxundi, J. I., Quemada, I. y Ruiz-Ruiz, R. (2007). Trastornos del comportamiento en daño cerebral adquirido. Acción Psicológica, 4(3), 110-113.

Seel, R. T., Kreutzer, J. S. y Sander, A. M.(1997). Concordance of patients' and family members' ratings of neurobehavioral functioning after traumatic brain injury. Archives of Physical Medicine and Rehabilotation, 78(11), 1254-1259.

Simmond, M. y Fleming, J. (2003). Reliability of the self-awareness of deficits interview for adults with traumatic brain injury. Brain Injury, 17(4), 325-337.

Soury, S., Mazaux, J. M., Lambert, J., De Seze, M., Joseph, P. A., Lozes-Boudillon, S.,... Levin, H. S. (2005). The neurobehavioral rating scalerevised: assessment of concurrent validity. $A n$ nales de Réadaptation et de Médecine Physique, 48(2), 61-70.

Stalder, F., Messerli, N., Hofer H., Frischknecht, E., Znoj, H. y Barth, J. (2013). Effect of psychological interventions on depressive symptoms in long-term rehabilitation after an acquired brain injury: a systematic review and meta-analysis. 
Archives of Psysical Medicine and Rehabilitation, 94(7), 1386-1397.

Vanier, M., Mazaux, J. M., Lambert, J., Dassa, C. y Levin, H. S. (2000). Assessment of neuropsychologic impairments after head injury: interrater reliability and factorial and criterion validity of the Neurobehavioral Rating Scale-Revised. Archives of Physical Medicine and Rehabilotation, 81(6), 796-806.

Waldron, B., Casserly, L. M. y O'Sullivan, C. (2013). Cognitive behavioural therapy for depression and anxiety in adults with acquired brain injury: what works for whom? Neuropsychological Rehabilitation, 23(1), 64-101.

Warriner, E. M. y Velikonja, D. (2006). Psychiatric disturbances after traumatic brain injury: neurobehavioral and personality changes. Current Psychiatry Reports, 8(1), 73-80.

Williams, W. H., Evans, J. J., Fleminger, S. (2003). Neurorehabilitation and cognitive-behaviour therapy of anxiety disorders after brain injury: an overview and a case illustration of obsessive compulsive disorder. Neuropsychological Rehabilitation; 13, 133-148.

Wilson, B. A. (1997). BADS: Behavioural assessment of the dysexecutive sindrome. USA: Thames Valley Test Company.

Wilson, B. A. (1999). Case studies in neuropsychological rehabilitation. USA: Oxford University Press.
Wilson, B. A. (2002) Towards a comprehensive model of cognitive rehabilitation. Neuropsychological Rehabilitation, 12(2), 97-110.

Wilson, B. A., Evans, L., Emslie, R. y Malinek, V. (1997). Evaluation of Neuro Page: a new memory aid. Journal of Neurology, Neurosurgery, and Psychiatry, 63, 113-115

Wise, K., Ownsworth, T. y Fleming, J. (2005). Convergent validity of self-awareness measures and their association with employment outcome in adults following acquired brain injury. Brain Injury, 19(10), 765-775.

Wood, R. L. y Alderman, N. (2011). Applications of operant learning theory to the management of challenging behavior after traumatic brain injury. Journal Head Trauma Rehabilitation, 26(3), 202-211.

Ylvisaker, M, Hanks, R. y Johnson-Green, D. (2002). Perspectives on neuropsychological rehabilitation of individuals with cognitive impairment after brain injury. Journal of head trauma rehabilitation, 17, 191-209.

Ylvisaker, M. (1998). Traumatic Brain Injury rehabilitation: children and adolsecents. Boston: Butterworth-Heinemann.

Ylvisaker, M., Jacobs, H. y Feeney, T. (2003). Positive supports for people who experience behavioural and cognitive disability after brain injury. Journal of Head Trauma Rehabilitation, 18, 7-32. 
